Alexander Refsum Jensenius* and Victoria Johnson ${ }^{\dagger}$

*University of Oslo, Department of Musicology PB 1017 Blindern

0315 Oslo, Norway

${ }^{\dagger}$ Norwegian Academy of Music

PB 5190 Majorstuen

0302 Oslo, Norway

a.r.jensenius@imv.uio.no,post@victoriajohnson.no

www.arj.no, www.victoriajohnson.no

\title{
Performing the Electric Violin in a Sonic Space
}

\begin{abstract}
This article presents the development of the improvisation piece Transformation for electric violin and live electronics. The aim of the project was to develop an "invisible" technological setup that would allow the performer to move freely on stage while still being in full control of the electronics. The developed system consists of a video-based motion-tracking system, with a camera hanging in the ceiling above the stage. The performer's motion and position on stage is used to control the playback of sonic fragments from a database of violin sounds, using concatenative synthesis as the sound engine. The setup allows the performer to improvise freely together with the electronic sounds being played back as she moves around the "sonic space." The system has been stable in rehearsal and performance, and the simplicity of the approach has been inspiring to both the performer and the audience.
\end{abstract}

How can a performer use the body to control live electronics? This is probably one of the most discussed and explored topics in the world of computer music performance today. Our approach has been to reduce and simplify the technological setup used in performance, which has resulted in greater artistic and expressive freedom.

The starting point for the work being presented here was the idea of letting the performer (the second author) "navigate" in a large soundscape on stage. As an electric violinist, she has been playing with electronics for a long time, enjoying the possibilities of wireless audio transmission and not having to worry about sound feedback (as an acoustic violinist playing into a microphone would). As she started to explore various types of sound effects and processing, however, the growing collection of effects pedals, MIDI controllers, and computers led to the need for cables and a physical (and visual) presence of various technologies in performance. The aim of this project was to explore how she could be liberated from the technology (and cables) and move freely in space again.

All the pieces that the performer has developed over the last few years have been sonically dense with a highly visual presence on stage (www.victoriajohnson.no). We therefore wanted to create a minimalist piece with sparse use of

Computer Music Journal, 36:4, pp. 28-39, Winter 2012

(c) 2013 Massachusetts Institute of Technology. electronics and little visual clutter, one that allowed the performer to improvise freely while wirelessly controlling the electronics herself. The end result is the improvisation piece Transformation for electric violin and live electronics.

From our initial discussions, several research questions appeared: How can we create an "invisible" technological setup that still allows for a fine control of the live electronics? How can we create an electronic part that allows for interaction possibilities that are musically interesting for the performer? What type of sound processing or creation can be used so that the performer feels in control of the whole sound palette during performance?

Our aim in this article is to present and evaluate the artistic research and development process that led to the piece Transformation. Through such a critical reflection and discussion of our own practice, we also hope to contribute to the discourse on artistic practice in general (Borgdorff 2006). But first we will start by presenting some background information on the technological challenges that the performer has explored over the years and that inspired the current project's focus on simplicity.

\section{Background}

The starting point for our collaboration was the performer's wish to be liberated from a technological 
setup that forced her to be stationary during performance. Being a classical violinist by training and profession, she has turned to performing mainly with electric violin over the last 10 years. This transition was based on a desire to explore a larger sonic palette than is possible with an acoustic violin, as well as to explore other musical genres. The electric violin is a feedback-free starting-point for working with live electronics. There are many different types of electric violins: MIDI violins, multi-pickup violins, etc. In this project we have been using a traditional four-string electric violin with a regular built-in pickup.

The performer's main focus has been on exploring a large set of extended performance techniques on the electric violin, many of which are explained by Strange and Strange (2001). It is interesting to note here that many well-known extended techniques for the acoustic violin may be extended further when performed on an electric violin, and even further when combined with live electronics. An example of such techniques is bowing on the tailpiece, pegs, and body of the instrument, which, combined with distortion effects or strong reverberation, open new and exciting soundscapes. Techniques that work well with delay and granulation effects include the use of crush-tones, using metal parts on the bow to produce sound, and bowing directly on the bridge or behind the bridge.

Over the years, the performer has explored several different strategies for controlling live electronics: (1) effects pedals and foot controllers, (2) handand bow-based controllers, (3) machine-listening systems, (4) motion capture, and (5) video analysis. All of these techniques have their benefits and challenges, which we will discuss in the following sections.

\section{Performing with Effects Pedals and Foot Controllers}

There are many positive aspects of using guitar pedals with electric violin: They are easily available and easy to use, having standardized settings and a long tradition in the electric guitar world. With the right combination of pedals, it is possible to explore a large sound palette and many musical genres.

Some performers, including Kimura (2003), have argued that using pedals may have an impact on the violin technique and may be distracting for the audience. This, however, depends entirely on how the pedals are used and how seamlessly the use of the pedals is integrated with the rest of the performance. After ten years of performing both composed and improvised music with various types of pedals, the second author feels that the pedals have become an integrated part of her extended instrument. They require no extra cognitive load during performance, and they integrate well with the various pieces she has developed. Another important aspect of performing with pedals, as opposed to other types of controllers, is that they free up the hands to carry out regular violin performance actions.

There are, however, some challenges when performing with pedals. For example, some pedals give a clicking sound when changing settings, which is often a larger distraction than the visual effect of pressing the pedal. Second, having a large set of pedals calls for a cluttered and complex setup. Third, most pedals are based on effects processing, i.e., they are sound-modifying and not sound-producing.

Some of the drawbacks of effects pedals may be overcome by instead using general-purpose (MIDI) foot controllers connected to a hardware sampler or synthesizer, or to a computer. Although such a setup can be potentially limitless, the performer has felt that it is actually more restrictive than using dedicated pedals, because the addition of a computer makes the total amount of gear more complex, both physically and virtually.

\section{Performing with Hand-Based Controllers}

Several research projects have focused on bow-based violin control over the last decade: Bossa (Trueman and Cook 2000), Hyperbow (Young 2002), the IRCAM bow (Bevilacqua et al. 2006), the NOTAM bow (Guettler, Wilmers, and Johnson 2008; Wilmers 2009), the commercially available K-Bow (McMillen 2008), the hand sensors used for the Overtone violin (Overholt 2005), and the Augmented Violin Glove 
(Kimura et al. 2012). Although there are certainly many interesting aspects of performing with such sensors, our experience with two of them (the NOTAM bow and the K-Bow) has also shown some technological and conceptual problems that we will discuss in the following paragraphs.

\section{Construction}

Adding electronics to a bow changes its weight and hence its tipping point. Quantitatively these changes are not very large, but for a skilled performer the extra weight is noticeable and requires adjustment while playing. Adjusting the technique for a specific bow is possible, but not ideal if changing between bows in performance. Also, adding fragile electronics to the bow reduces the performance possibilities of the performer.

\section{Technology}

Many wireless devices use Bluetooth for communication, benefiting from easy connectivity and broad support in software and hardware. Unfortunately, we have found that Bluetooth is not (yet) truly reliable for musical performance, with unexpected communication dropouts and too long reconnection time. For this reason, we and others have been exploring ZigBee for musical applications in general (Torresen, Renton, and Jensenius 2010; Fléty and Maestracci 2011; Torresen et al. 2012), and for violin performance in particular (Kimura et al. 2012). These ZigBee-based devices seem to overcome some of the current challenges of Bluetooth devices, particularly those of a stable data flow and reliable connection (and reconnection) with the host computer.

\section{Interpretation}

Even with a reliable wireless communication, interpreting and mapping data from inertial sensors is not a straightforward process. For example, although accelerometer data give seemingly useful information, they only indirectly represent physical acceleration. Second, estimating absolute position based on inertial sensors is not trivial, as evidenced by the drift seen in even the most advanced commercial systems (Skogstad and Nymoen 2011). Thus, thorough calibration (and recalibration) is necessary to ensure a stable system in performance.

\section{Concept}

Although it is certainly fascinating to track the bow's motion and position, we are not convinced that it is artistically interesting. For a violinist, the bowing is already the most important part of her regular performance technique. Even when using seemingly "transparent" machine-learning techniques, e.g., the Gesture Follower (Bevilacqua et al. 2007), our experience is that the performer starts using the bow differently when she knows it is being used for parameter control.

\section{Machine-Listening Systems}

Kimura (2003) argues that real-time sound analysis may be a better way to capture information about the performance than tracking using a sensor bow. We have tested some different types of machinelistening systems, primarily based on tracking pitch (using fiddle [Puckette, Apel, and Zicarelli 1998]) and some basic spectral features (using analyzer [Jehan 2005]). The positive side of such systems is that they allow for touchless control of the system, albeit only when the performer is producing sound. One challenge with machine-listening systems is the precision of the tracking, especially for complex soundscapes, e.g., with multiple instruments and/or polyphonic sound material. This, however, can be overcome with machine-learning techniques and some musical knowledge in the algorithms. Although we will certainly consider using machinelistening in the future, we did not feel it was suitable for the current project.

\section{Motion Capture}

Motion-capture technologies are becoming increasingly popular in interactive systems. Whereas motion capture may be defined as any type of system that tracks information about a person's position 
Figure 1. An overview of

the developed system. The

boxes with dotted lines

show the non-real-time

parts.

and motion in space over time, we will use it here to denote systems that can track full-body motion.

We have tested several different full-body motioncapture systems to see if they could be useful for our project. Using an infrared optical motioncapture system (Qualisys) was abandoned early in the process. Such systems provide for accurate, precise, and fast tracking of absolute position, but they are not an option for smaller performance setups. Even though there exist affordable systems (e.g., the Optitrack system from NaturalPoint), the large amount of equipment needed (cameras, stands, cables), calibration challenges, etc., makes it too complex to tour with for a single musician. Therefore, an inertial-based motion-capture system would be a better solution. Here we have tested the Xsens MVN motion-capture suit, a commercially available system that can capture absolute position based on inertial sensor fusion (accelerometers, gyroscopes, and magnetometers). This is a fully integrated on-body system which is transportable in a medium-sized suitcase, and which provides high speed, accuracy, and precision, as well as wireless connectivity. As such, it solves many of the problems that are found with optical infrared systems, but it also introduces some new ones: reliability of the wireless communication, dependency on batteries, and inaccuracies of the absolute position due to drift. The biggest problem with such a suit, however, is that the suit itself is quite heavy and uncomfortable to wear. Also, the visual presence of the suit makes the technology very apparent, even when trying to hide it under regular clothing.

\section{Video Analysis}

Testing various motion-capture systems, we realized that what they could offer was not along the artistic lines that we were interested in pursuing: minimalism in both music and technology. This led us to test video analysis as a motion-capture technique. Video analysis is slower and less precise than all the sensing systems presented earlier. The big advantage with video analysis, however, is that it can be entirely unobtrusive to the performer. Also, video analysis can easily be used to track position

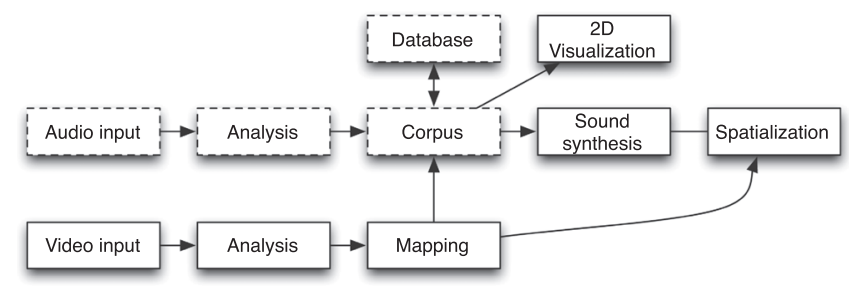

in space, although at a coarser level than, say, an infrared motion-capture system.

One of the biggest challenges when working with video analysis in a performance context is to separate the foreground from the background. This is particularly problematic when creating a setup that should be moved between different concert venues of various sizes and layouts, with different lighting conditions, and so on. Our solution has been to place the camera in the ceiling, so that only the stage floor is captured. This is an easy solution to avoid problematic backgrounds, e.g., uncontrolled light or moving audience members. Also, using a grayscale image as the source material for the analysis makes the setup more robust against changing stage lights. As will be discussed in the next section, such a setup has met our requirements of being easy to set up, reliable under many conditions, and having no cables on the body of the performer.

\section{Setup}

Figure 1 presents an overview of the technological setup we have developed for the piece Transformation. It is based on a pre-recorded and pre-analyzed database of sounds, real-time video analysis, position to sound mapping, and sound spatialization. The following sections will describe the three main parts of the setup: video tracking, sound engine, and sound spatialization.

\section{Video Tracking}

For the video tracking we used some of the videoanalysis modules implemented in the Musical Gestures Toolbox (Jensenius, Godøy, and Wanderley 
2005) for the open framework Jamoma for Max (Place and Lossius 2006). Three different video modules are used for getting video from the camera, calculating the motion image (the running frame difference), and using this as the basis for finding the area and center position of the motion image. Then we know the location of the performer in space, and how much she moved.

The first author has used and improved these video modules for analysis and performance over many years, and they have proven to be very stable and reliable under all sorts of performance conditions. Although it is not the most advanced computer vision method around, the advantage of using a simple tracking method is that it is CPUfriendly and requires no calibration except for simple threshold adjustments. So the video analysis patch runs comfortably together with sound processing patches on a normal laptop.

\section{Sound Engine}

The aim of the sound part of the piece was to allow the performer to control the playback of pre-recorded electric violin sounds, so that she could improvise with "herself." We started our exploration of the sound part by recording a large set of material covering the broad range of sonic possibilities that extended techniques on a violin can offer. This material was then used to test different sound-processing techniques together with the motion-capture system.

Our first experiment was based on distributing sound files linearly in space, so that the performer could "scrub" through the sounds when moving on stage, similar to what has been explored in the Embodied Generative Music project (Peters 2010). The intuitive connection between location on the floor and the sound being played back gave the performer a sense of being in direct control of the sound by merely walking through the room, and she would also start to remember exactly where the different sonic objects were located in space. But the setup was also limiting and did not feel enough like an instrument to the performer. First, the spatial and temporal resolution of the video tracking $(640 \times 480$ pixels at $25 \mathrm{fps})$ was not sufficient to give the performer a sense of fidelity in the interaction with the sonic space. Second, the interaction felt one-dimensional, because she could only scrub through the files in one dimension (back and forth). Third, we did not find a good conceptual solution for turning sound playback on and off when she was moving in and out of the captured space.

In the second set of experiments we divided the space into an $8 \times 8$ grid in which the performer could play back certain sounds by entering into a zone in the grid. Here the interaction with the sound material was experienced as more interesting to the performer, but we did not find a satisfactory way of controlling the duration of the samples being played back. It was easy to start playing back the samples when she moved into a new zone in the grid, but it was trickier to find a solution for when sounds should stop playing, especially if she was moving back and forth between two or more zones. So although this setup was more interesting to improvise with than the first setup, it still did not work musically the way we wanted.

The third method we explored was concatenative synthesis, using the CataRT (Schwarz et al. 2006) and FTM (Schnell et al. 2005) libraries for Max. CataRT is based on cutting up a collection of sound material into small sonic fragments, each of which are analyzed using a set of low-level audio features: pitch, loudness, periodicity, spectral flatness, etc. The final result is a database containing pointers to each of the original sound files, the start and stop position of each segment, and the results for all the extracted features. These features are then used for plotting the relative distance between the sound fragments in a 2-D display. This display can then be used as the starting-point for playing back individual sound fragments, by copying the relevant part of the sound file to the output buffer and applying short fade-ins and fade-outs to avoid clicks in the playback. The playback mode can be chosen in the engine: playing individual fragments, looping the same fragment, or looping over adjacent fragments. All in all, this makes it possible to create a large variety of sonic textures that can easily be controlled by moving around in the 2-D display. 
We quickly discovered that mapping the 2-D position in space to the 2-D control space of CataRT was very intuitive for the performer. Also, because sounds in CataRT are grouped according to sonic qualities, it is possible to import a large set of sound files and have CataRT group similar sound fragments close to each other. This makes for a rich and nuanced sonic space that can be used for improvisation.

After experimenting with different types of sound material, we came up with a sample library of approximately ten minutes of different violin sounds (mainly pizzicato and flageolets), which is used as input to the system. In CataRT we use a window size of $250 \mathrm{msec}$, a duration that is perceptually relevant but still short enough to allow splicing sounds together. We have found that a sonic distribution with "spectral centroid" on one axis and "periodicity loudness" on the other is the most interesting combination for interacting with our sound database. This setting gives the performer (and the audience) a clear perceptual understanding of the two axes, while still allowing interesting sounds to appear close to each other on the floor. We prefer the "fence" triggering method, which only triggers a sound fragment when the performer gets close to its position in the 2-D display, and which does not trigger or retrigger any fragments if she is standing still between groups of sonic objects.

\section{Speaker Setup and Sound Spatialization}

The last part of our system is the spatialization of sounds in space. Because the piece focuses on exploring the physical space through a virtual space, we also wanted to distribute sound in space depending on where the performer was moving.

For the most recent performances of Transformation, the room has been set up with chairs on all four sides of the stage, a set of smaller speakers at the corners of the stage area, and four larger speakers on the diagonals close to the walls. A simple one-to-one mapping was set up between location on the floor and the placement of sounds, so that the sound would move in space with the performer. This was achieved using the CPU-friendly vector-based
Figure 2. A sketch drawn

at one of our first

rehearsals of

Transformation. The

sketch shows the speaker

setup and the imagined

path of the performer

through the sonic space.

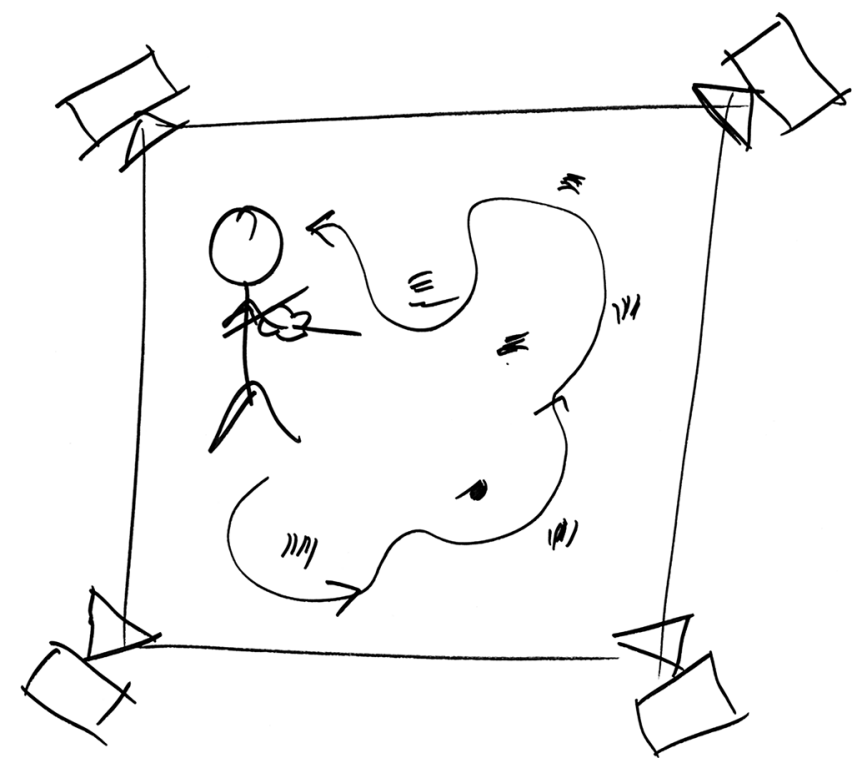

amplitude panning (VBAP) sound-spatialization technique (Pulkki 2001).

Because there is no audible acoustic sound from the electric violin, we have explored different solutions for projecting the violin sound in performance: through all speakers; through a single, separate speaker; or spatialized with the sounds from the computer. We have found the latter to be most successful, because it makes the connection between physical location of the instrument and actual sounding result more obvious. Even though all performances of the piece have been carried out in fairly reverberant spaces, we have still found the need to add a little extra reverb to make a more holistic soundscape of the sound fragments being played from CataRT.

\section{The Piece}

The piece Transformation is improvised for each performance based on a loose plan of how the performer should move through the sonic space on stage. There is no score for the piece, but a sketch of a typical performance is shown in Figure 2. The piece can be seen as what Dean (2003, p. xiii) calls a referent-based improvisation, in which the path through the space is the referent. Although the 
Figure 3. Rehearsal before the performance at the National Library of Norway, 4 February 2010. Here eight small speakers were set up at the front of the stage, and a subwoofer was placed on the left side.
Figure 4. Rehearsal before the performance at the Norwegian Academy of Music, 3 September 2010. The camera hangs from the ceiling, and eight loudspeakers are placed in two squares around the square stage area.

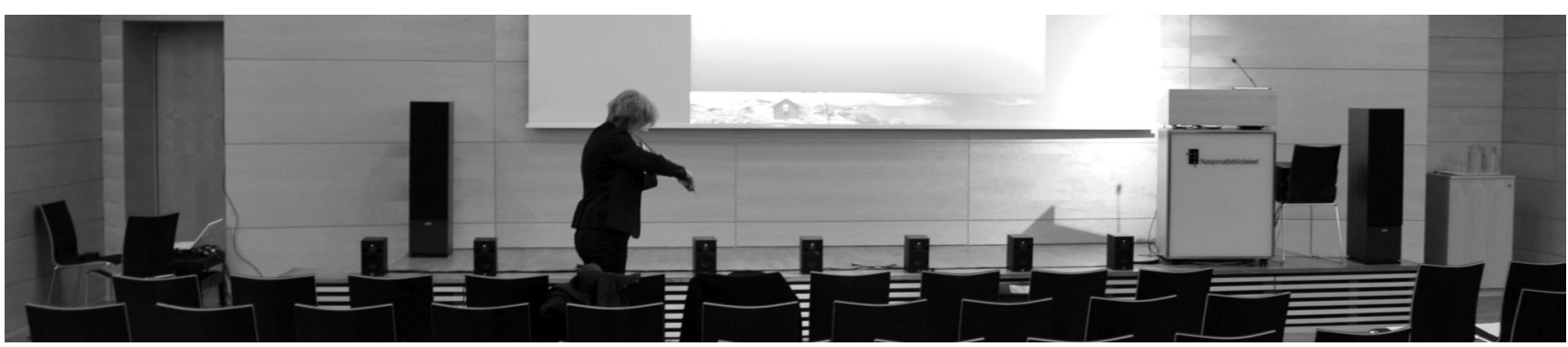

Figure 3

path is planned, the piece is highly reliant on the interplay between the performer and the sounds coming from the computer during the performance.

\section{Performances}

The piece has so far been presented in four public performances. The first of these were in the foyer of the Norwegian Opera and Ballet on 26 November 2009. Here we performed as part of a larger setup, and with sonic material from bells and water drops. The setup consisted of five speakers placed in a row against the ceiling, and both authors participated in the sonic exploration of the space in performance. Still in an early stage of development, this performance was a first test to check that the setup worked properly, was easy to set up, intuitive to play with, and interesting for the audience.

The second public performance was at the National Library of Norway on 4 February 2010, this time with eight speakers in a row at the front of a small stage (see Figure 3). Due to constraints of the performance space, the interaction with the sonic space became mainly one-dimensional. On the positive side, we found that our newly recorded violin sounds worked well.

The third performance was at the Norwegian Academy of Music on 3 September 2010 (see Figure 4). Here we set up two sets of four speakers. Four smaller speakers were placed at the corners of the defined stage area. These speakers were facing towards the performance space so that the performer could hear the sounds, but also because we wanted to make the audience experience the sounds as coming

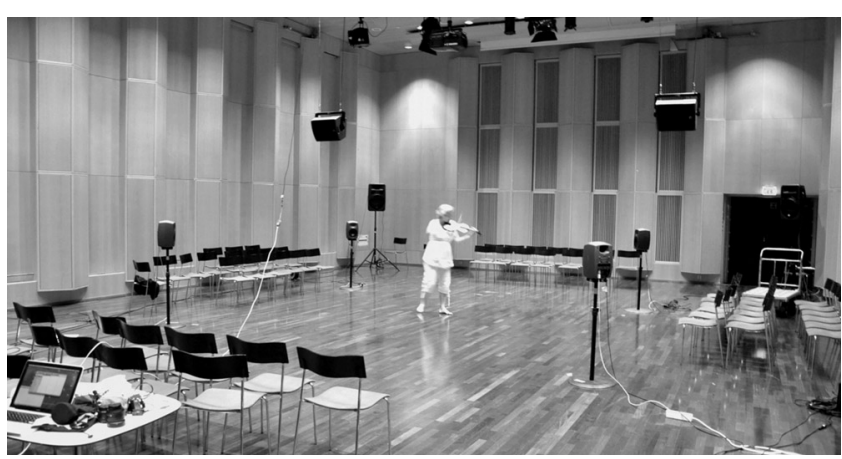

Figure 4

from "within" the space. Four larger speakers were placed at the outer corners of the room, and were used mainly to add low-frequency content to the sound mix.

During performance a white dance carpet and white lighting was used to create a visual "emptiness" in the physical space, and thereby enhance the presence of the sounds in space (see Figure 5). This was the first performance where we got close to our initial aims of a visually and sonically minimalist piece. Because the tracking and interaction worked so well, however, it became apparent that the sound material could be improved.

The fourth performance was on 28 March 2011 at the Norwegian Academy of Music. For this performance we recorded a new set of sounds with a subtle, sustained quality: flageolets and various types of bowing on the bridge and on the body of the instrument. The performance setup was otherwise similar to the third performance, but with one important change: This time the 
Figure 5. From the concert of 3 September 2010. The white carpet was used to mark the boundaries of the performance space.
Figure 6. From the concert of 28 March 2011. Here the stage was lifted so that the white square became more visible.

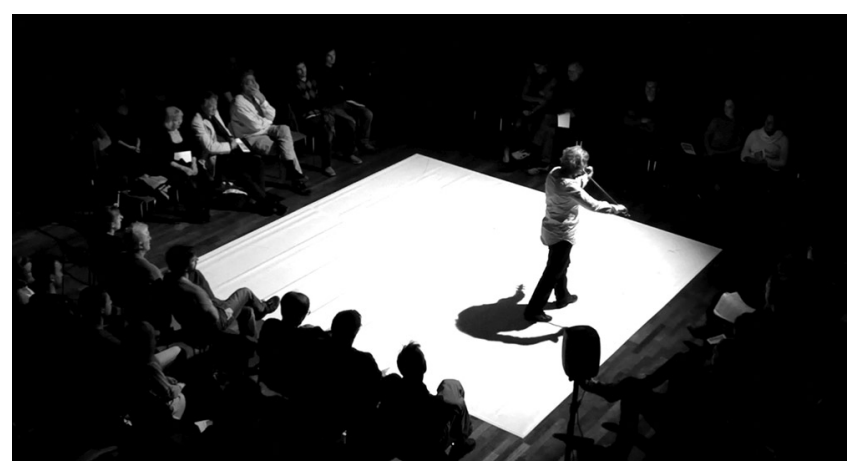

Figure 5

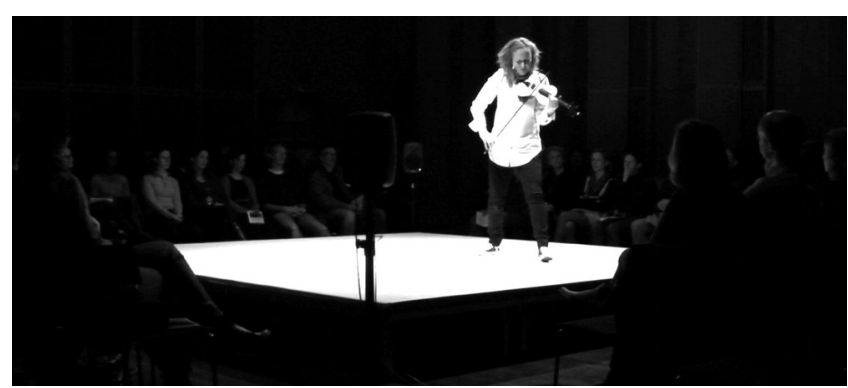

Figure 6

white dance carpet was placed on a $50-\mathrm{cm}$ tall stage platform (see Figure 6). This made the white square "hang" in space, and it also helped to make the performer more visible to the audience (www.youtube.com/watch?v=JTk7vZsj7Fc).

\section{Evaluation}

The piece Transformation is the result of an artistic development process in which we have explored a cycle between development of the technologies; recording and selecting sonic material; musical exploration in workshops, and public performances; and critical reflections on the whole process. In the reflection we have mainly been focusing on three aspects: technology, interaction, and sonic output.

\section{Technology}

The evaluation of the technological setup can be summarized in the following points.

\section{Rigging}

We have created a setup that could be taken on tour, and which should therefore be easy to rig up and down. For our current solution, the most time-consuming part is to mount the camera in the ceiling, which should take less than 15 minutes if a ladder or stage lift is available.

\section{Stability}

The software system uses three core components: Jamoma, CataRT, and VBAP for Max. All of these have been extensively tested and used in performance by a number of people for many years. We have not experienced any instability issues in workshops or performances.

\section{Usability}

The software has been developed so that the performer can set up and run the performance herself. When turned on, the system does not require any attention or control besides general sound level adjustments by a sound technician.

\section{Scale}

The setup scales well to different rooms. The main challenge of the tracking is to get a large enough image, which is based on a combination of the ceiling's height and the camera lens. The audio setup also scales well, and we have been testing different types of speaker constellations in performance. As such, the technical setup and conceptual idea can be easily adjusted and scaled to different venues and settings.

\section{Interaction}

The possibilities of interaction can be seen as a combination of the following technological and artistic elements. 


\section{Freedom}

The setup has given the performer the freedom to do what she wanted musically, and has liberated her from standing or sitting still. She has cablefree and reliable control of the electronics during performance, and is free to interact with the entire sound palette. We use pre-recorded sound material, but there are no pre-determined musical structures. The system thus resembles an instrument that can be played and improvised with for long periods of time.

\section{Complexity}

A core challenge when developing the system was to create a setup that was complex enough to be sonically interesting for a full improvisation piece. Our initial testing with laying out sound files in space did not have the complexity we were looking for. One reason for this was the limited resolution of the tracking; hence the mapping to sound did not result in the preferred level of detail in the interaction. Using CataRT with a distribution of hundreds of short sound fragments, we find that there is a good balance between complexity and simplicity.

\section{Creativity}

Because the system picks short fragments from a large sound collection it is impossible to predict exactly what type of sound material will appear. This element of surprise, within clear boundaries, is highly valued by the performer, and gives her creative freedom to improvise with the system.

\section{Reproducibility}

Although it is important to have a system that is creative and complex, it is also important to know how it will respond (Kimura 2003). In that sense, CataRT's distribution of sounds based on perceptual features has proven to be of vital importance. The performer knows where different sonic qualities can be found on the floor, and this allows her to move around the space and discover (or rediscover) various sound objects.

\section{Dimensionality}

The current tracking solution is very simple, based only on movement and position on the floor. As discussed earlier, we have tested a number of other types of control systems, all of which have more output parameters. Our experience, however, is that the current reduced model works better for us, becasue it allows for more intuitive interaction. The performer is already spending most of her focus on playing the violin, and has limited "cognitive bandwidth" available for controlling the computer. Having to work in more dimensions would interfere with the sound-producing actions on the violin. As such, moving in a 2-D space has made it possible for her to continue performing with her traditional violin technique, while still being able to control the electronics simply by moving on the floor.

\section{Sonic Output}

The final sound of the piece is based on the merging of the direct sound from the electric violin and the violin sounds played from the computer.

\section{Violin}

Our experience, and also that of others (e.g., as reported by Palacio-Quintin 2008), has shown that sensors or controllers may interfere with traditional performance technique. Our "controllerfree" system allows the performer to play the electric violin using her existing violin technique. The result is better sound in performance.

\section{Recorded Sound}

Over the course of the project we have recorded new sound material several times, based on an increased understanding of what works well with the system. Because all the sounds are taken from the violin, the sonic result could easily have been that of a string "orchestra." This has not been the result, though, 
mainly because the selected sound files were based on various types of extended techniques exploring the extremities of the sonic possibilities of the instrument. That said, we found it very inspiring to work with a system where it was possible for the performer to play along with material that was either similar or contrasting to the sound she was producing herself. This type of sonic transparency made for a freer interaction between musician and computer.

\section{Fragmented Sound}

Because the sound material was based on short sound fragments, it does not easily allow for creating longer sustained phrases. But by moving through the space it is possible to generate "phrases" of fragmented sounds, and connect them together while playing on top of them.

\section{Form}

The piece is improvised, without a score, but is based on a coarse path we have defined through the space. The path defines the beginning and end, as well as various sonic qualities that the performer will move through during the piece, but is otherwise open to exploration.

\section{Discussion}

Besides the specific points mentioned in the previous section, the development process has also resulted in some general thoughts about how it is possible to work and play with live electronics.

\section{Simplicity}

Our experience of performing with electronics for more than ten years each has shown us that we often use technologies and develop setups that are too complex. This leads to performances that may be confusing to the audience, and sometimes even to the performer(s). For that reason, we were interested in pursuing a strategy of simplicity throughout the entire project: "simplicity is about subtracting the obvious, and adding the meaningful" (Maeda 2006, p. 89). The development of Transformation has shown us that a seemingly "simple" setup can be very rewarding artistically, as it has given the performer freedom to move and improvise. Consequently, Transformation is a piece she always looks forwards to playing.

\section{Performing in Space}

Coming from classical and contemporary musical traditions, we are used to performing in traditional concert and chamber halls. In Transformation we have changed this by placing the bright, white stage in the middle of the audience. The stage becomes an "instrument" itself, a combination of physical, visual, and sonic space with which the performer can play. Due to this visual focus on the performance space, the performer's presence on stage is very much in attention, something that has made us reflect on the expectations with which the audience might meet the performer: Is she seen as a dancer or actress rather than a musician? The performer has no training in dance or theater, so we decided early on that she should perform as a musician, and that her movements on and around the stage should be purely motivated by that of producing sound, either electronically or on the violin. As long as this is shown clearly, we believe that the movements of a performer on stage are beautiful in themselves, albeit at a different aesthetic level than what would be expected from a dancer or actress.

\section{Performing in the Air}

A challenge of using video analysis in interactive systems is the lack of resistance and feedback in the "instrument." In our current setup this felt less problematic, because the electronic sound was so tightly connected to the physical location in the performance space. After rehearsing for a few hours at a time, the performer developed an embodied sensitivity of where to find certain sound objects. This made her able to walk directly to the location 
she wanted, and also choose a trajectory to the point that would fit with the musical ideas she had in mind.

\section{Future Work}

There are many possibilities for refinement and development of the system and piece that will be explored in future research, as follows.

\section{Tracking}

We will explore using high-speed and high-resolution cameras to improve the temporal and spatial resolution of the system. It may also be relevant to test other types of tracking solutions, but we will be careful about keeping the focus on simplicity in both technology and concept.

\section{Micro-level}

With more precise tracking it is possible to see whether very subtle movements, what can be called micromovements, may be used to control the system. We have received audience feedback on the perceived tension when the performer stands still or moves very little. It will be interesting to explore this type of tension further with more precise tracking.

\section{Visuals}

The last two performances of Transformation have been highly visual, focused on the white, bright square in the stage area. Although we like the simplicity of the current setup, we will also test adding some subtle live visuals on the floor.

\section{Localized Sound}

In previous performances the sound of the electric violin has been spatialized together with the electronic sound. We want to see how a ceiling mounted speaker may be used to project the sound of the violin from above. Such a separation between the violin and the electronic sounds may be positive, but it may also remove some of the current blending between the two layers of sound.

\section{Live Sampling}

Rather than start with pre-recorded sound files, we will start with an empty sonic space and then gradually fill it with recorded sounds as the performer moves and plays in the space.

\section{Quietness}

One of the most important challenges, though, is to ensure that the piece remains peaceful, quiet, and contemplative.

\section{Acknowledgments}

Thanks to the anonymous reviewers and the $C M J$ editors for feedback. The project has been supported by the Norwegian Artistic Research Program, Norwegian Research Council, Norwegian Academy of Music, and University of Oslo.

\section{References}

Bevilacqua, F., et al. 2007. "Wireless Sensor Interface and Gesture-Follower for Music Pedagogy." In Proceedings of the International Conference on New Interfaces for Musical Expression, pp. 124-129.

Bevilacqua, F., et al. 2006. "The Augmented Violin Project: Research, Composition and Performance Report." In Proceedings of the International Conference on New Interfaces for Musical Expression, pp. 402-406.

Borgdorff, H. 2006. The Debate on Research in the Arts. In Proceedings of Sensuous Knowledge: Focus on Artistic Research and Development, pp. 1-31.

Dean, R. T. 2003. Hyperimprovisation: ComputerInteractive Sound Improvisation. Middleton, Wisconsin: A-R Editions, Inc.

Fléty, E., and C. Maestracci. 2011. "Latency Improvement in Sensor Wireless Transmission using IEEE 802.15.4." In Proceedings of the International Conference on New Interfaces for Musical Expression, pp. 409-412.

Guettler, K., H. Wilmers, and V. Johnson. 2008. "Victoria Counts-A Case Study with Electronic Violin Bow." 
In Proceedings of the International Computer Music Conference, pp. 569-662

Jehan, T. 2005. "Creating Music By Listening." PhD thesis, Massachusetts Institute of Technology.

Jensenius, A. R., R. I. Godøy, and M. M. Wanderley. 2005. "Developing Tools for Studying Musical Gestures within the Max/MSP/Jitter Environment." In Proceedings of the International Computer Music Conference, pp. 282-285.

Kimura, M. 2003. "Creative Process and Performance Practice of Interactive Computer Music: A Performer's Tale." Organised Sound 8(3):289-296.

Kimura, M., et al. 2012. "Extracting Human Expression for Interactive Composition with the Augmented Violin." In Proceedings of the International Conference on New Interfaces for Musical Expression, pp. 99-102.

Maeda, J. 2006. The Laws of Simplicity: Design, Technology, Business, Life. Cambridge, Massachusetts: The MIT Press.

McMillen, K. A. 2008. "Stage-Worthy Sensor Bows for Stringed Instruments." In Proceedings of the International Conference on New Interfaces for Musical Expression, pp. 347-348.

Overholt, D. 2005. "The Overtone Violin." In Proceedings of the International Conference on New Interfaces for Musical Expression, pp. 34-37.

Palacio-Quintin, C. 2008. "Eight Years of Practice on the Hyper-Flute: Technological and Musical Perspectives." In Proceedings of the International Conference on New Interfaces for Musical Expression, pp. 293298.

Peters, D. 2010. "Enactment in Listening: Intermedial Dance in EGM Sonic Scenarios and the Bodily Grounding of the Listening Experience." Performance Research 15(3):81-87.

Place, T., and T. Lossius. 2006. "Jamoma: A Modular Standard for Structuring Patches in Max." In Proceedings of the International Computer Music Conference, pp. 143-146.
Puckette, M. S., T. Apel, and D. Zicarelli. 1998. "RealTime Audio Analysis Tools for Pd and MSP." In Proceedings of the International Computer Music Conference, pp. 109-112.

Pulkki, V. 2001. "Spatial Sound Generation and Perception by Amplitude Panning Techniques." PhD thesis, Helsinki University of Technology.

Schnell, N., et al. 2005. "FTM-Complex Data Structures for Max". In Proceedings of the International Computer Music Conference, pp. 9-12.

Schwarz, D., et al. 2006. "Real-Time Corpus-Based Concatenative Synthesis with CataRT." In Proceedings of the International Conference on Digital Audio Effects, pp. 279-282.

Skogstad, S. A. v. D., and K. Nymoen. 2011. "Comparing Inertial and Optical MoCap Technologies For Synthesis Control." In Proceedings of Sound and Music Computing, pp. 421-426.

Strange, P., and A. Strange. 2001. The Contemporary Violin: Extended Performance Techniques. Berkeley, California: University of California Press.

Torresen, J., E. Renton, and A. R. Jensenius. 2010. "Wireless Sensor Data Collection Based on ZigBee Communication." In Proceedings of the International Conference on New Interfaces for Musical Expression, pp. 368-371.

Torresen, J., et al. 2012. "Development and Evaluation of a ZigFlea-Based Wireless Transceiver Board for CUI32." In Proceedings of the International Conference on New Interfaces For Musical Expression, pp. 409-412.

Trueman, D., and P. R. Cook. 2000. "Bossa: The Deconstructed Violin Reconstructed." Journal of New Music Research 29(2):121-130.

Wilmers, H. 2009. "Bowsense-An Open Wireless Motion Sensing Platform." In Proceedings of the International Computer Music Conference, pp. 287-290.

Young, D. 2002. "The Hyperbow: A Precision Violin Interface." In Proceedings of the International Computer Music Conference, pp. 489-492. 